\title{
Biological Effects on C. Elegans in the Application of Essential Oils as Natural Fungicides
}

\author{
Miriam Zanellato ${ }^{1}$, Priscilla Boccia ${ }^{1}$, Maria Grazia Berardinelli ${ }^{1}$, Lucia Donnarumma ${ }^{2}$, Elena Sturchio ${ }^{\mathbf{1}}$ \\ ${ }^{1}$ Department of Technological Innovation and Safety of Plants, Product and Anthropic Settlements (DIT), Italian Workers' \\ Compensation Authority (INAIL), Rome, Italy \\ m.zanellato@inail.it; \\ p.boccia@inail.it; m.berardinelli@inail.it; e.sturchio@inail.it ; \\ ${ }^{2} 2$ Consiglio per la Ricerca in Agricoltura e l'Analisi dell'Economia Agraria (CREA)- Centro di Ricerca Difesa e \\ Certificazione (DC), Rome, Italy. \\ lucia.donnarumma@crea.gov.it
}

\begin{abstract}
Essential oils due to their antimicrobial properties, low persistence and toxicity are useful in agriculture in order to reduce the use of pesticides for healthier and sustainable crop production systems. The synergistic effect obtained by combining different essential oils allowed to reduce the concentrations of single oils, therefore the potentially toxic components, maintaining and improving the efficacy against parasites. Our previous studies demonstrated the efficacy of different mixtures of essential oils as natural fungicides, a dose dependent effect and a decrease of the genotoxic effect of three oil mixtures compared to the mixtures of two oils. Our work aims to evaluate if the use of essential oils mixtures can increase the mortality or influence the fertility of Caenorhabditis elegans nematodes, exposed to different mixtures of essential oils (previously tested) to assess the safety use of these mixtures on not-target organisms. Preliminary results demonstrated a lower C.elegans mortality in the three-oil mixture compared to the two-oil mixture, confirming that combining different essential oils allows reducing the potential toxicity effect. It is important to improve these studies in order to reduce and/or substitute the use of pesticides with natural compounds for the highest environmental benefits and for human health.
\end{abstract}

Keywords: Essential oils, natural fungicides, genotoxicity assays, C.elegans, pest management.

\section{Introduction}

In the past decade, the legislation concerning the use of plant protection products, in particular pesticides, has undergone a thorough review, aimed to protect the human health and the environment by reducing their impact.

The Directive (EC) 128/2009 established a Community framework for the sustainable use of pesticides and it proposed, among other things, to provide a pest management of agricultural crops with a lower use of plant protection products, in order to safeguard a high level of biodiversity and the protection of the biotic adversities of plants.

The new regulatory framework for plant protection products (PPPs) laid out in Commission Regulation (EC) No 1107/2009 and Commission regulation (EU) No 283/2013 explicitly requires consideration of impacts on non-target species, on their ongoing behaviour and on biodiversity and the ecosystem, including potential indirect effects via alteration of the food web [1]. In this regards the Goals 15 of Sustainable Development launched by ONU for the 2030 agenda specifies that microorganisms and invertebrates are key factors to ecosystem, but their contributions are still poorly known and rarely acknowledged [2].

Following the review of plant protection products and the entry into force of Regulation (EC) 1107/2009 for their marketing, the availability of fungicides has decreased, moreover the Directive (EC) 128/2009 promoted approaches to crop protection to reduce the use of plant protection products [3].

Currently, there is an increasing interest in using natural substances as alternative strategies to pesticides in agriculture, because of their evident advantages such as biodegradability, low persistence in the environment, not bioaccumulation.

However, in order to evaluate the introduction and the use of a natural substance in common phytoiatric practices some aspects should be considered, in particular the proposed substance must satisfy some properties: efficacy against the target organism, safety and biological selectivity, standardized composition and formulation, ready availability. Moreover, as well 
as synthetic pesticides, any natural substance in order to obtain authorization and registration required a series of toxicological data.

Among the natural substances proposed for pest management, essential oils are excellent candidates due to their potential antimicrobial, fungicide and herbicide properties. Essential oils generally have lower persistence and toxicity and a lower risk of resistance development to pathogenic microorganisms compared to synthetic pesticides $[4,5]$.

Moreover combining different essential oils with different properties, it is possible to obtain mixtures of essential oils that show a greater effectiveness against the different parasites compared to a single essential oil; this allows thus the reduction of the single oils concentrations and therefore the potentially toxic components (synergistic effect) [6].

About that, since a decade our studies have been aimed to the applicability of essential oils as natural fungicides. In our preview studies, we evaluated the use of single essential oils and essential oils mixtures (two or three oils) against the cucurbit powdery mildew pathogen Podosphaera fusca (synonym Podosphaera xanthii), in zucchini plants (var. Romanesco) cultivated in greenhouse, as unique treatments or alternated with synthetic fungicides as maintaining strategy $[7,8]$. We also assessed the potential genotoxicity and phytotoxicity of these oils by laboratory testing and any residuals in soils using the in vivo model system (Vicia faba), because it is important to know all the potential biological effects and chemical changes caused by their uses in agriculture $[9,10]$.

Our results highlighted the efficacy of the two and three essential oils mixtures against Podosphaera fusca, with a very satisfactory disease control achieved in term of disease incidence and disease severity reduction, without any phytotoxic effect in treated plants after the compounds application [8]. The two different mixture tested demonstrated a dose-dependent activity and the three oils mixture is effective and less phytotoxic compared to the single oil and the two oils mixture due to the lower concentration of single component $[9,10]$.

In the present work we wanted to investigate if or how much the use of essential oils mixtures tested in our preview studies can increase the mortality or influence the fertility of Caenorhabditis elegans nematodes, natural inhabitants of soil that may be a not-target organisms affected by phytoiatric practises. We evaluated the biological effects of three of the most efficacy essential oils mixtures previous tested by solid-killing assay and fertility bioassays; this in vivo model system provides an useful tool for toxicity tests, thanks to its unique characteristics, as well as constant number of cells in all larval stages, transparent body, short average life span and very simple cultivation and maintenance.

\section{Materials and Methods}

\subsection{Essential Oils Mixtures Preparation}

The tested essential oils mixtures are constituted by three different essential oils: rosemary oil (R), clove oil (C) and oregano oil (O). These essential oils was collected from the local market in Rome (Italy) and their composition was previously analysed by chromatographic techniques (Table 1) [7, 11].

Table 1: Percentage of main components in essential oils..

\begin{tabular}{|l|l|c|c|c|}
\hline Essential oils & $\alpha$-pinene $\%$ & 1,8 -cineole $\%$ & Terpinen-4-ol \% & Thymol \% \\
\hline Rosemary oil & 7,3 & 36,9 & & \\
\hline Clove oil & & & 5,8 & \\
\hline Oregano oil & & & & 40,1 \\
\hline Essential oils & $\alpha$-pinene $\%$ & 1,8 -cineole $\%$ & Terpinen-4-ol \% & Thymol \% \\
\hline Rosemary oil & 7,3 & 36,9 & & \\
\hline Clove oil & & & 5,8 & \\
\hline
\end{tabular}

Oils were emulsified with $0.05 \%$ Tween $20[12,13]$ before their application. We tested three essential oils mixtures, that in greenhouse previous studies demonstrated the greatest efficacy as fungicides, one constituted by two essential oils (BIS - clove plus rosemary) and two constituted by three essential oils (TRIS / 1/2 TRIS - clove, rosemary plus origanum) (Table 2). 
Table 2. The composition of the essential oil mixtures tested.

\begin{tabular}{|c|l|}
\hline Treatment & Composition \\
\hline BIS & $0,8 \mathrm{ml} /$ L Clove \\
& $1,6 \mathrm{ml} /$ L Rosemary \\
\hline \multirow{2}{*}{ TRIS } & $0,8 \mathrm{ml} /$ L Clove \\
& $1,6 \mathrm{ml} /$ L Rosemary \\
& $0,4 \mathrm{ml} /$ L Origanum \\
\hline \multirow{2}{*}{$1 / 2 \mathrm{TRIS}$} & $0,4 \mathrm{ml} /$ L Clove \\
& $0,8 \mathrm{ml} /$ L Rosemary \\
& $0,2 \mathrm{ml} /$ L Origanum \\
\hline
\end{tabular}

\subsection{C.elegans Toxicity Assays}

Wild-type nematodes (N2 strain provided by the CGC) were cultivated in Petri dishes containing Nematode grow media (NGM) agar and a food source consisting of E.coli OP50 grown as superficial monolayer. In order to perform toxicity tests, the nematodes were synchronized in order to obtain a colony of individuals at the same larval state [14].

Solid killing assay

We evaluated whether the treatment could cause a shortening of the nematode lifespan, for example caused by accidental exposure, and therefore a higher mortality. Multi well plates containing $1 \mathrm{ml}$ of NGM per well were prepared; $100 \mu \mathrm{M}$ of ampicillin to avoid bacterial contaminations and 40mM of 5-Fluoro-2'-deoxyuridine (FUDR) to obtained sterile nematode was added to NGM agar, before pouring it into the multi well plates. $50 \mu 1$ of E.coli OP50 liquid culture were seeded into each well and incubated overnight at $37^{\circ} \mathrm{C}$, in order to obtain a bacterial monolayer. Subsequently, $50 \mu 1$ of the three essential oils mixtures (BIS, TRIS, $1 / 2$ TRIS) were added to each well of the dedicated plate, while no treatment was applied to the negative control dedicated plate. About 40 synchronized L4/young adult nematodes were transferred into the multi well plates (four nematodes for well) and scored for dead or alive daily, for 25 days, by gently touching them with a platinum wire (Fig. 1). Three independent experiments were conducted ( 100 L4/young adult nematodes tested for each treatment). Statistical analysis by Logrank test (Mantel-Cox) was performed.

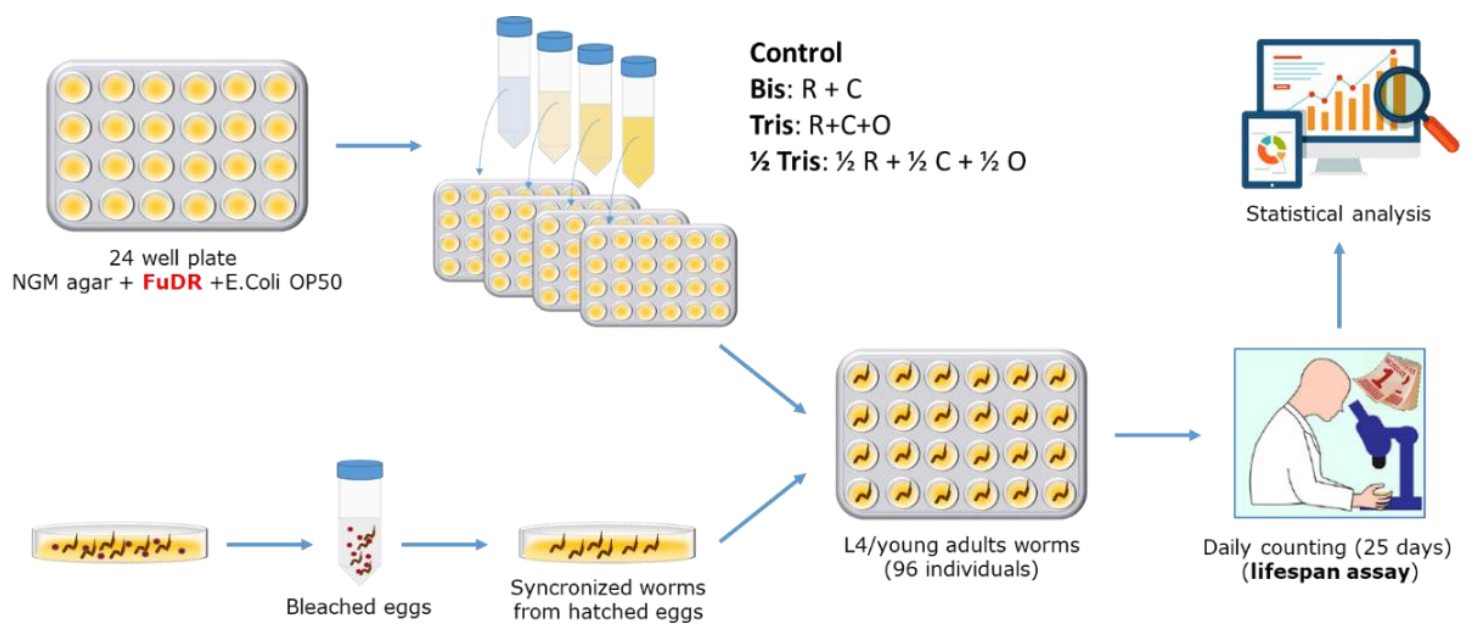

Fig. 1. Solid killing assay. 


\section{Fertility assay}

We evaluated whether the treatment could interfere with the fertility of nematodes in term of decrease in offspring. well plate containing $1 \mathrm{ml}$ of NGM per well were prepared and 50 $\mu 1$ of E.coli OP50 liquid culture were seeded into each and incubated overnight at $37^{\circ} \mathrm{C}$, in order to obtain a bacterial monolayer. Subsequently, $50 \mu$ of the three essential oils mixtures were added to each well of the dedicated plate, while no treatment was applied to the negative control dedicated plate.

About 30 synchronized L4/young adult nematodes were transferred into the multi well plates (one nematode for well); every day the nematodes were transferred in a new well and the eggs and the offspring were counted for $72 \mathrm{~h}$ (Fig. 2).

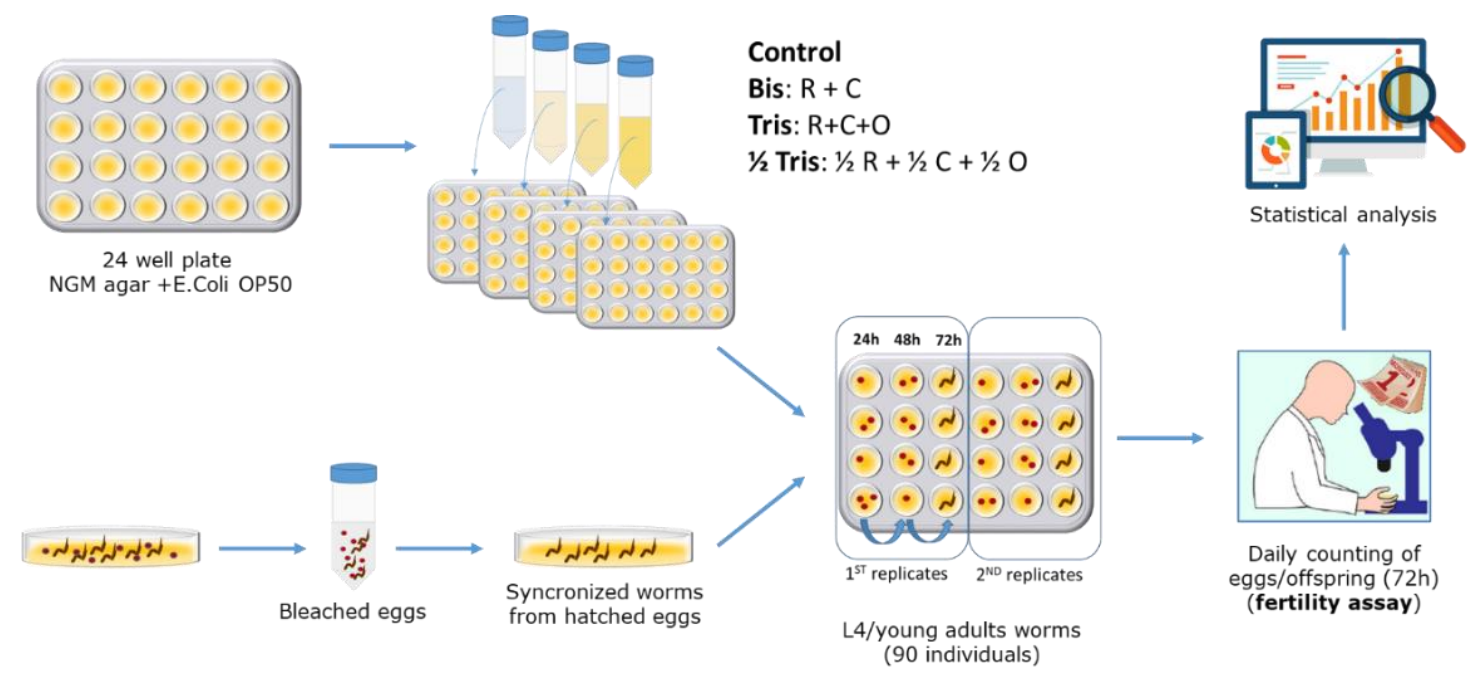

Fig. 2. Fertility assay.

Statistical analysis by one way ANOVA (Tukey's test) was performed to determine the significances of differences between samples and non-treated controls. Statistical significance was accepted for $\mathrm{p}$ values $<0.05$, and significant changes are indicated by asterisks in figures.

\section{Results}

Solid killing assay

We considered a more appropriate approach to study the C.elegans lifespan in the solid culture medium assay, in order to be as close as possible to a real exposure condition of the non-target organisms, living on soil, after the crop treatment with essential oils in the field.

The obtained results showed that BIS and TRIS treatments caused a significative shortening of the nematodes lifespan. As shown in the figure 3, B) and D), the two essential oil mixture treatments caused the total population death at the day 19. At the same time the $1 / 2$ TRIS treatment showed no significative differences in nematode lifespan compared to the control. 

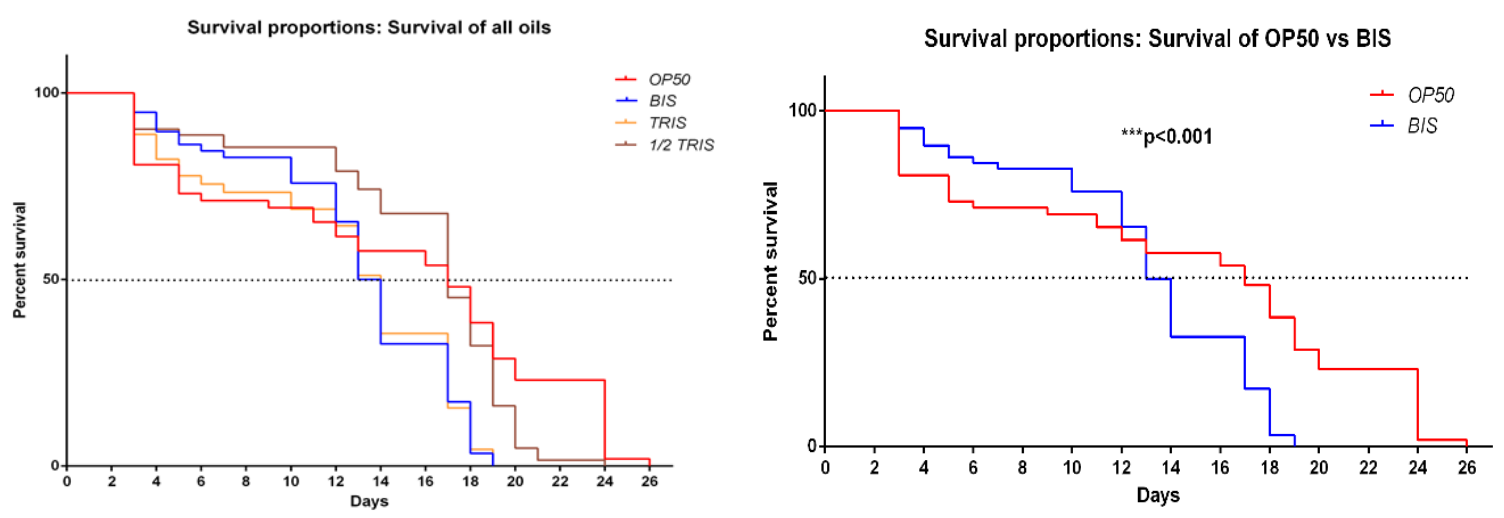

A)

B)
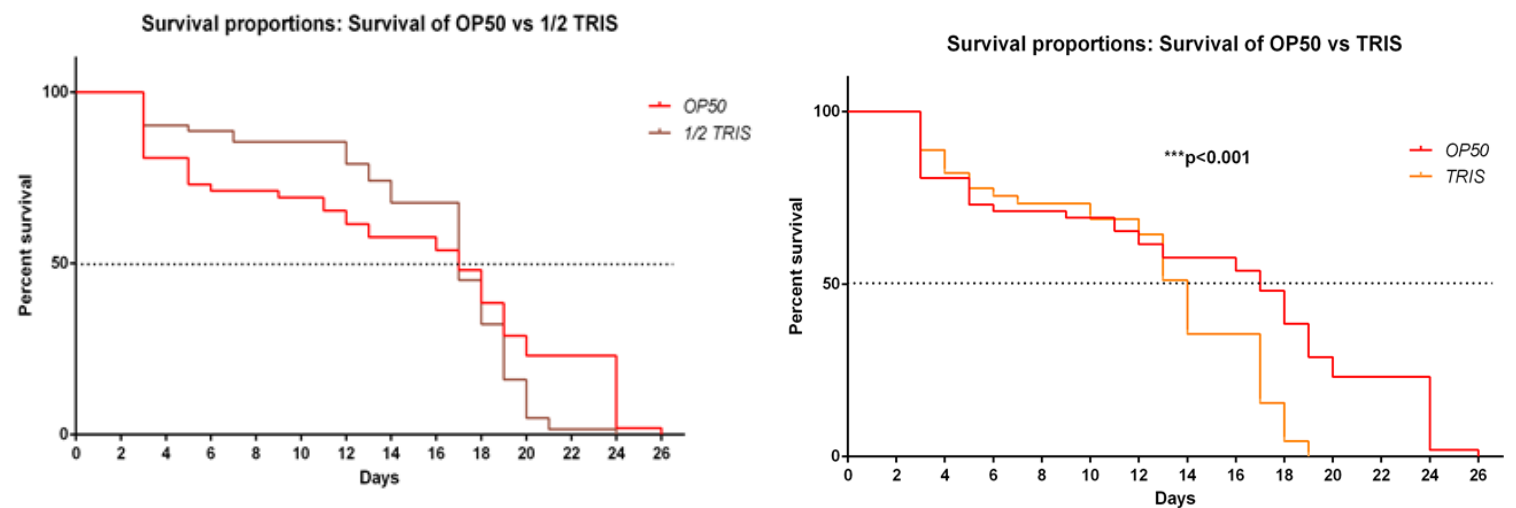

C)

D)

Fig. 3. Solid killing assay results. A) cumulative data; B) BIS compared to the negative control; C) TRIS compared to the negative control; C) $1 \frac{1}{2}$ TRIS compared to the negative control.

We also observed in a primarily phenotypic analysis during the "censoring" step [15] an higher number of worms that exhibit "protruding vulva" (the intestine protrudes from the vulva) or undergo "bagging" (matricidal hatching wherein the larvae hatch inside the mother and it dies), in the C.elegans population exposed to TRIS treatment (Fig. 4).
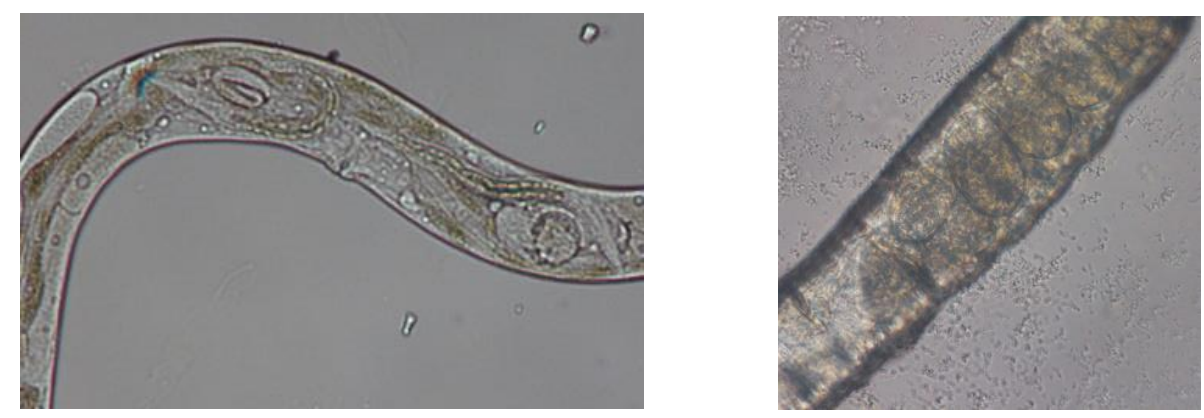

Fig. 4. Worms that exhibit "protruding vulva" and undergo "bagging". 


\section{Fertility assay}

The fertility assay was performed counting daily the amount of eggs layed and the offsprings, but we considered interesting to focus our attention on the amounts of offsprings, as an indicator of a possible interference of treatment on variations of density of nematode population on soil.

As shown in figure 5, the TRIS treatment resulted in a significant decrease of offsprings after the exposure. The BIS and $1 / 2$ TRIS treatments seem to not affect C.elegans fertility showing a offspring amount that is almost similar to that of control treatment.

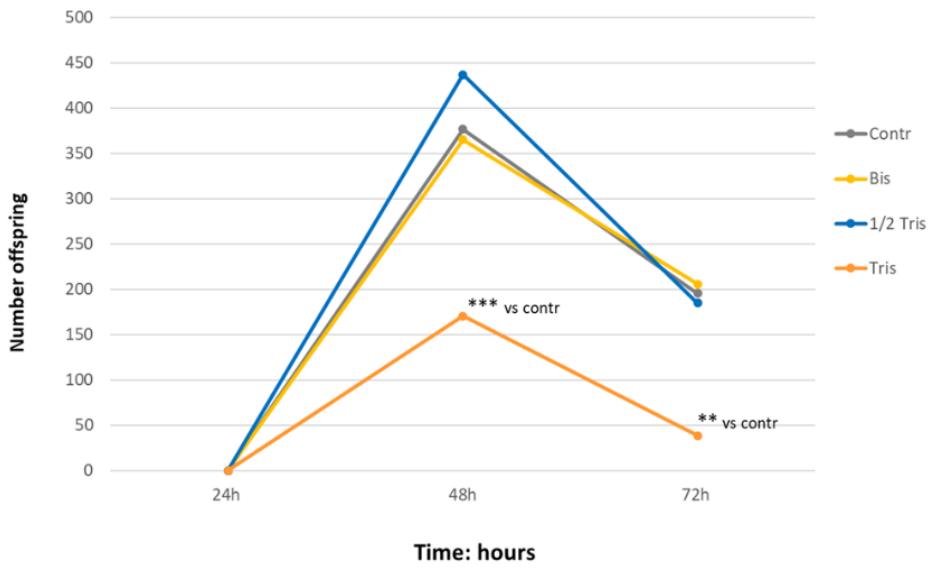

Fig. 5. Fertility assay results.

\section{Conclusions}

The use of essential oils based mixtures provides the opportunity to limit the use of synthetic products and then the reduction of the risks of their distribution in the environment and on the edible part of the crops, as well as the possibility to distance the intervals of treatment with oils since they can protect the crop longer. It is also important to underline their usefulness in order to reduce the risk of developing pathogen resistance phenomena, typical of synthetic products. Therefore, having available potential products that ensure a satisfactory level of protection at low risk of resistance induction, is a valid alternative [16].

In previous studies we demonstrated a good efficacy of the tested essential oils mixtures as natural fungicides both as single treatment, as well as alternated with a synthetic fungicide and the effectiveness against Podosphaera fusca of the three oil mixture, even when it was used at half concentration (synergistic effects of the three essential oils). We did not observe any residual toxicity in soil. A dose dependent activity of the tested essential oils mixtures was also demonstrated.

The present work represents a further step towards the global knowledge of the action mechanisms of essential oils, essential for their practical application in agriculture.

Our results highlight that the $1 / 2$ TRIS mixture seems not interfere in C.elegans lifespan, compared to BIS and TRIS treatments containing a double dose of clove oil and rosemary oil, probably because their anthelmintic properties are more appreciable $[17,18]$.

The fertility assay shows a dramatic decrease of offspring after the TRIS treatment, compared to the BIS and $1 / 2$ TRIS treatments. Therefore we can hypothesize that this effect could be due to the higher concentration of oregano essential oil. 
In vivo studies performed by Hollenbach et al. [19] demonstrated that oregano essential oil can interfere in sperm and hormonal parameters, causing infertility.

The efficacy against the pathogen, the absence of phytotoxicity, the non-interference with non-target organisms lifespan lifespan and fertility demonstrated by $1 / 2$ TRIS mixture validates our hypothesis that by combining different essential oils it oils it is possible to obtain a good pest control, reducing at the same time the concentration of the single essential oils components and therefore the potentials toxic effects.

No health policy today can ignore the complex network of relationships between human, animals and environment health. The "One Health" approach interprets and takes into account the complexity of the connections between these three "actors", considering the complex relationships that govern the emergence of new infectious diseases, the role of environmental factors and climate change in terms of availability and healthiness of food, the impact of anthropogenic pressure on biodiversity and ecosystem health, the consequences of the exploitation of natural resources and the sustainability of food production [20].

Inevitably, humans change the ecosystems, being part of it, but at the same time we have the opportunity, by our choices, to affirm diversity or devalue it.

Essential oils are promising active compounds, because of their antimicrobial and anthelmintic properties that could be used in the development of effective alternative products, due to the real advantages in food safety and the low environmental impact. Nevertheless, further studies regarding the right doses and safety of essential oils should be carried out.

\section{References}

[1] European Food Safety Authority (EFSA). Scientific Opinion addressing the state of the science on risk assessment of plant protection products for non-target arthropods. EFSA Panel on Plant Protection Products and their Residues (PPR) 2,3 European Food Safety Authority (EFSA), Parma, Italy EFSA Journal 2015;13(2):3996

[2] United Nations Development Programme. GOAL 15 TARGETS @2019 United Nations Development Programme. http://www.undp.org/content/undp/en/home/sustainable-development-goals/goal-15-life-on-land/targets.html

[3] L. Donnarumma, F. Milano, S. Trotta, M.T. Schiavi, T. Annesi., "Risultati preliminari sull'utilizzo di oli essenziali per la difesa dello zucchino dall'oidio. Integrated protection strategies against zucchini powdery mildew with formulates based on essential oils", Proceedings of the Congresso Nazionale della Società Italiana per la Ricerca sugli Oli Essenziali. Roma, 15-17 novembre, 2013, http://www.natural1.it/fitoterapia//item/1369-atti-del-1-congresso-nazionale-della-siroeparte-2.

[4] D.J. Daferera, B. N. Ziogas and M. G. Polissiou. "GC-MS Analysis of essential oils from some greek aromatic plants and their fungitoxicity on Penicillium digitatum", J. Agric. Food Chem., n. 48, pp. 2576-2581, 2000.

[5] M. B. Isman and C. M. Machial, "Pesticide based on plant essential oils: from traditional practice to commercialization", In: Advances in Phytomedicine, Naturally Occurring Bioactive Compounds, Rai M., Carpinella M.C., Cap. 2, Oxford, Elsevier B. V. (eds), 2006, pp 29- 44.

[6] Y. J. Fu, Y. G Zu, L. Y. Chen, X. G. Shi, Z. Wang, S. Sun and T. Efferth, "Antimicrobial activity of clove and rosemary essential oils alone and in combination”, Phytother. Res., n. 21, pp. 989-994, 2007.

[7] E. Sturchio, L. Donnarumma, T. Annesi, F. Milano, L. Casorri, M. Masciarelli, M. Zanellato, C. Meconi and P. Boccia, "Essential oils: an alternative approach to management of powdery mildew diseases", Phytopathol. Mediterr. Vol. 3, n. 53, pp.385-395, 2014. doi: 10.14601/Phytopathol_Mediterr-13607.

[8] L. Donnarumma, E. Sturchio, F. Milano, P. Boccia, M. Zanellato and T. Annesi, "Effectiveness of Essential Oils Mixtures Based on Soy Emulsifier Against Powdery Mildew on Zucchini Plants", J. Plant. Physiol. and Pathol., 5:4. 2017. doi:10.4172/2329-955X.1000169

[9] E. Sturchio, M. Mecozzi, P. Boccia, C. Meconi, M. Zanellato and L. Donnarumma, "Genotoxic effects of different mixtures of essential oils", Proceedings of 5th International Conference on Environmental Management, Engineering, Planning and Economics (CEMEPE) and SECOTOX Conference, Mykonos Island, Greece, June 14-18, 2015. 
[10]E. Sturchio, P. Boccia, M. Zanellato, C. Meconi, L. Donnarumma, G. Mercurio and M. Mecozzi, "Molecular and structural changes induced by essential oils treatments in Vicia faba roots detected by genotoxicity testing", J. Toxicol. Environ. Health A, 2016. DOI: 10.1080/15287394.2015.1124059.

[11] L. Donnarumma, F. Milano, S. Trotta and T. Annesi "Essential oils against zucchini powdery mildew", J. Phytopath., n. 163, pp. 877-885, 2015. doi: 10.1111/jph.12387. Blackwell Verlag GmbH.

[12] M. Reuveni, V. Agapov, R. Reuveni, "Controlling powdery mildew caused by Sphaerotheca fuliginea in cucumber by foliar sprays of phosphate and potassium salts", Crop Prot., n. 15, pp. 49-53, 1996.

[13] V. Terzi, C. Morcia, P. Faccioli, G. Valè, G. Sacconi and M. Malnati, "In vitro antifungal activity of the tea tree (Melaleuca alternifolia) essential oil and its major components against plant pathogens", Lett. in Appl. Microbiol., Vol. 6, n. 44, pp. 613-618, 2007.

[14] T. Stiernagle, "Maintenance of C.elegans", In C. elegans: A Practical Approach, ed. I. A. Hope, Oxford: Oxford University Press., 1999, pp. 51-67.

[15] F. R. G. Amrit, R. Ratnappan, S.A. Keith, A. Ghazi, “ The C. elegans lifespan assay toolkit”, Methods, n. 68, pp. 465475, 2014.

[16] T. Annesi, F. Milano, P. Pulcini, A. Matere and L. Donnarumma, "Strategie integrate di difesa dall'oidio dello zucchino con formulati a base di oli essenziali", Giornate Fitopatologiche, n.2, pp. 567-574, 2016.

[17] M.A. Zoral, K. Futami, M. Endo, M. Maita, T. Katagiri, "Anthelmintic activity of Rosmarinus officinalis against Dactylogyrus minutus (Monogenea) infections in Cyprinus carpio", Vet. Parasitol., n. 247, pp. 1-6, 2017. doi: 10.1016/j.vetpar.2017.09.013. Epub 2017 Sep 14.

[18] V. G. Charitha, J. Adeppa and P. Malakondaiah, "In vitro anthelmintic activity of Syzygium aromaticum and Melia dubia against Haemonchus contortus of sheep", Short communication, Indian J. Anim. Sci., vol. 8, n. 87, pp. 968-970, 2017. [19] C. B. Hollenbach, R. Scheer Bing, R. Stedile, F. Peixoto da Silva Mello, T.L. Schuch, M.R. Alves Rodrigues, F. Bastos de Mello and J.R. Braga de Mello, "Reproductive Toxicity Assessment of Origanum vulgare Essential Oil on Male Wistar Rats", Acta Sci. Vet., n. 43, p. 1295, 2015.

[20] P.M.G. Rabinowitz, M. Pappaioanou , K.L. Bardosh, L. Conti, "A planetary vision for one health", BMJ Glob Health., vol. 5, n. 5, e001137, 2018. doi:10.1136/ bmjgh-2018-001137. 